Research Paper

\title{
LncRNA EIF3J-AS1 functions as an oncogene by regulating MAFG to promote prostate cancer progression
}

\author{
Chen Ye ${ }^{*}$, Shengfei Qin*, Fei Guo*, Yue Yang, Huiqing Wang, Chao Zhang ${ }^{\bowtie}$, Bo Yang ${ }^{\bowtie}$ \\ Department of Urology, Shanghai Changhai Hospital, P. R. China, Shanghai 200433, China \\ * These authors have contributed equally to this work \\ $\triangle$ Corresponding authors: Chao Zhang, Postal address: 168 Changhai Road, Shanghai, 200433 China. Email: tony373@163.com. Phone: 86-13818119856. Bo \\ Yang, Postal address: 168 Changhai Road, Shanghai, 200433 China. Email: yangbochanghai@126.com. Phone: 86-13386277121 \\ (C) The author(s). This is an open access article distributed under the terms of the Creative Commons Attribution License (https://creativecommons.org/licenses/by/4.0/). \\ See http://ivyspring.com/terms for full terms and conditions.
}

Received: 2021.03.20; Accepted: 2021.10.23; Published: 2022.01.01

\begin{abstract}
Long non-coding RNAs (IncRNAs) can modulate various biological processes and behaviors in most human cancers. LncRNA EIF3J-ASI has been reported as an oncogene in various tumors, but whether it exerts functions in malignant progression and gene expression in prostate cancer (PCa) remains unknown. In this study, we investigated the high level of EIF3J-ASI in PCa tissues and cells, and used functional assays to show that knocking down EIF3J-AS1 inhibited PCa cell proliferation and metastatic ability. A preliminary mechanistic investigation also showed that EIF3J-AS1 may increase the expression of MAF bZIP transcription Factor G (MAFG) in PCa. The expression correlation between EIF3J-ASI and MAFG was found to be positive in PCa tissues. Finally, rescue assays showed that MAFG might be involved in the EIF3J-ASI-mediated malignant phenotype in PCa cells. This study demonstrated that EIF3J-ASI/MAFG may play a key role in facilitating PCa progression.
\end{abstract}

Key words: Prostate Cancer; lncRNA; EIF3J-AS1; MAFG

\section{Introduction}

Prostate cancer (PCa) is the most common malignant tumor and the second leading cause of cancer death among men worldwide [1]. Despite many studies that aimed to improve the diagnosis and treatment of $\mathrm{PCa}$, including PSA screening, PSMA theranostics, and new generation androgen deprivation therapy; the distant metastasis, castration resistance and neuroendocrine differentiation continue to limit long-term survival [2-5]. Exploring the underlying molecular mechanisms of these processed from different perspectives may help us better understand the tumorigenesis and progression of PCa.

LncRNAs are a class of non-noncoding RNAs that are greater than lengths 200 nucleotides. Studies have shown that lncRNAs play important roles in cancer biological processes, such as proliferation, apoptosis and metastasis [6, 7]. Recently, lncRNA
EIF3J antisense RNA 1 (EIF3J-AS1) was found to function as an oncogene in glioma, colorectal cancer, esophageal cancer, etc. [8-10], demonstrating its clinical potential as a biomarker and therapeutic target. However, the role of EIF3J-AS1 in PCa remains unclear. From the results of a past study, we demonstrated that EIF3J-AS1 is upregulated in PCa cells and tissues, and downregulation of EIF3J-AS1 inhibited cell growth and metastasis in PCa cells.

Previous research has demonstrated that lncRNAs can regulate gene expression through a variety of mechanisms, such as chromatin modification, transcriptional regulation and posttranscriptional regulation, by interacting with DNA, RNA and proteins [11-13]. How EIF3J-AS1 regulates which key genes in $\mathrm{PCa}$ influence tumorigenesis and malignant progression in $\mathrm{PCa}$ must be studied. In the results of analysis a past 
study, we found that EIF3J-AS1 is positively correlated with MAFG (MAF bZIP transcription Factor G) in PCa cells and tissues by analyzing a public database and performing sample validation. MAFG is a bZIP-type transcriptional regulator that can bind to DNA as a homodimer, leading to transcriptional repression owing to a lack of transactivation domains [14]. Previous studies have shown that MAFG contributes to the malignant progression of hepatocellular cancer and colorectal cancer $[15,16]$. In this study, we found that MAFG could promote cell proliferation and metastasis in $\mathrm{PCa}$, and downregulating MAFG can impair the cancer-promoting effects of EIF3J-AS1 in PCa. These findings described the basics of the cancer-promoting role and regulatory mechanism of the EIF3J-AS1/MAFG axis in PCa progression.

\section{Materials and methods}

\section{Tissues samples and cell lines}

All 36 paired PCa and matched adjacent healthy control (HC) tissues were obtained from the urology department of Shanghai Changhai Hospital between 2016 and 2019. All patients provided consent for the use of all samples, and the collection of human tissue samples was approved and supervised by the Ethics Committee of Changhai Hospital.

\section{Cell culture and transfection}

The human PCa cell lines PC-3, LNCaP, and DU-145 and the normal prostate epithelial cell line RWPE-1 were obtained from the American Type Culture Collection (ATCC). The PCa cell lines were cultured in RPMI 1640 medium supplemented with $10 \%$ fetal bovine serum (FBS, Gibco, USA). RWPE-1 cells were cultured in keratinocyte-SFM that was supplemented with epidermal growth factor and bovine pituitary extract (Gibco, USA). All cells were maintained at $37{ }^{\circ} \mathrm{C}$ in humidified air containing $5 \%$ $\mathrm{CO} 2$ according to the manufacturer's instructions. The siRNAs designed for EIF3J-AS1-AS1, MAFG and corresponding controls were all procured from GenePharma (Shanghai, China) for gene silencing. LV-NC and LV-MAFG for gene overexpression were also procured from GenePharma (Shanghai, China). Cell transfections were performed by using Lipofectamine 2000 (Invitrogen, U.S.A.) following to the manufacturer's instructions.

\section{Cell proliferation, migration and invasion assay}

Cells were plated in 96-well plates (2000/well). After $24 \mathrm{~h}, 48 \mathrm{~h}, 72 \mathrm{~h}$, and $96 \mathrm{~h}$ of incubation, cell viability was determined by Cell Counting Kit 8 assay (CCK8) with plate reader ELx800"T (BioTek, USA) according to the manufacturer's instructions.
Transwell assays were performed to determine cell migration and invasion ability. Starvation-treated (serum-free for $24 \mathrm{~h}$ ) cells were seeded $(5 \times 104)$ in the upper chamber of transwell inserts without or without Matrigel-coated membranes (Corning, USA) and cultured with serum-free medium. The lower chamber contained complete medium. After $48 \mathrm{~h}$ of incubation, the cells on the upper side of the membrane were scraped off, and the lower side cells were fixed with $4 \%$ paraformaldehyde and then stained with $0.1 \%$ crystal violet.

\section{RNA isolation and qRT-PCR}

Total RNA was extracted from cells or tissues using TRIzol Reagent (Invitrogen, USA), and cDNA was synthesized using the PrimeScript ${ }^{\mathrm{TM}}$ RT Kit (TaKaRa, Japan) following the manufacturer's protocol. Quantitative RT-PCR was performed using SYBR ${ }^{\circledR}$ Premix Ex Taq $^{\mathrm{TM}}$ (TaKaRa, Japan) in a StepOne-plus Real-Time PCR system (ABI, USA). The relative expression of EIF3J-AS1 and MAFG was calculated by the $2-\Delta \Delta \mathrm{Ct}$ method.

\section{Western blot assay}

All cells were collected and lysed in RIPA lysis buffer. Protein concentrations were detected by BCA assay (Thermo Scientific, USA) following the manufacturer's protocol. Proteins were separated by SDS/PAGE and transferred to PVDF membranes. Then, the membranes were incubated in blocking buffer for $1 \mathrm{~h}$ and incubated with primary antibody overnight. On the second day, the membrane was washed in PBS three times and incubated with secondary antibody for $1 \mathrm{~h}$. The primary antibodies used were MAFG (Abcam, ab154318) and $\beta$-actin (Abcam, ab8226).

\section{Statistical analysis}

Statistical analyses were performed with SPSS 17.0 , and numerical data are shown as the mean \pm SD of three independent experiments. Paired Student's $t$ tests, one-way ANOVAs, and Pearson correlation analyses were performed for comparisons, and a $\mathrm{pA} P$ value $\left({ }^{*} \mathrm{p}<0.05\right.$; ** $\mathrm{p}<0.01$; ** $\left.\mathrm{p}<0.001\right)$ was considered significant.

\section{Results}

\section{EIF3J-ASI is upregulated in PCa tissues and cells}

To investigate the role of EIF3J-AS1 in PCa, we first measured the expression of EIF3J-AS1 in 36 paired PCa and matched adjacent HC tissues by qRTPCR. The expression level of EIF3J-AS1 was significantly upregulated in PCa tissues compared with HC tissues (Figure 1A). Consistently, EIF3J-AS1 
was upregulated in DU145, PC3 and LNCaP PCa cell lines compared with the normal prostate epithelial cell line RWPE-1 (Figure 1B). Considered together, these findings suggest that EIF3J-AS1 may play an important role in the progression of PCa.
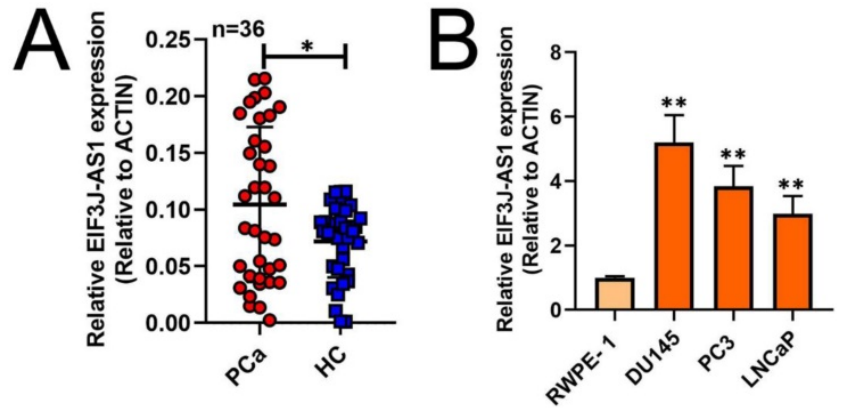

Figure 1. EIF3J-AS1 is upregulated in PCa cells and tissues. A and B, qRT$P C R$ assessed EIF3J-AS1 expression in PC tissues and control normal tissues, as well as in PC cells (DU145, PC3 and LNCaP) and the human normal prostate epithelial cell line RWPE-1. *P $<0.05$, ** $\mathrm{P}<0.01$, *** $\mathrm{P}<0.001$

\section{Downregulation of EIF3J-AS1 inhibits cell growth and invasion in PCa cells}

Based upon the above clinical data described above, we therefore performed several in vitro assays to describe the biological relevance of EIF3J-AS1 in PCa. Because PCa cells had higher levels of EIF3J-AS1, we treated these cells with EIF3J-AS1 knockdown (Figure 2A). In the CCK-8 assay, we observed that EIF3J-AS1 siRNA treatment markedly inhibited cell proliferation in PC3 and DU145 cells (Figure 2B). We also evaluated the invasion ability of control cells and EIF3J-AS1-knockdown PCa cells. In transwell migration and invasion assays, we observed that EIF3J-AS1 siRNA treatment markedly inhibited cell migration and invasion in PC3 and DU145 cells (Figure 2C and D). Collectively, results showed that EIF3J-AS1 contributes to cell growth and invasion in PCa cells.
A
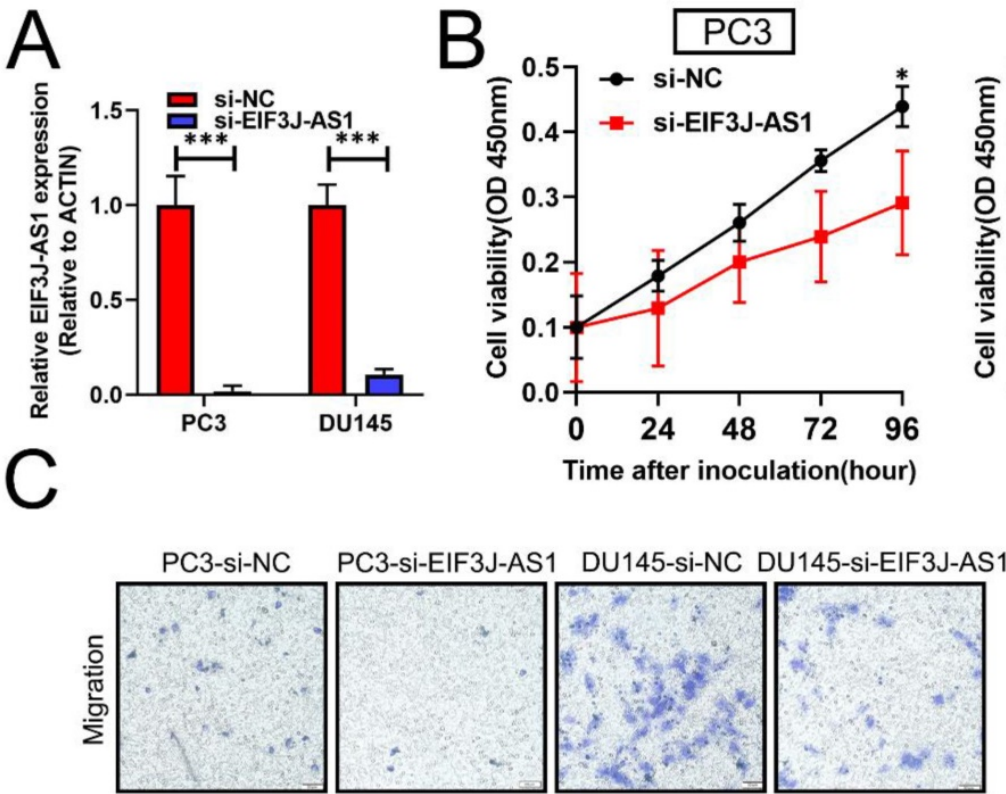

D
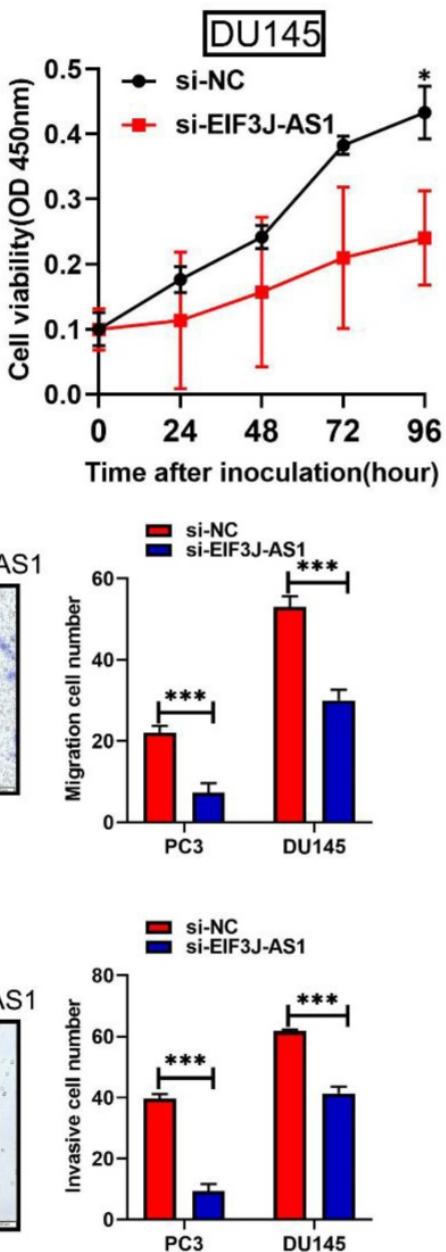

Figure 2. Downregulation of EIF3J-AS1 inhibits cell growth and invasion in PCa cells. A, qRT-PCR was used to assess EIF3J-AS1 expression in the indicated treated $\mathrm{PC}$ cell lines. B, The proliferation of si-EI3]-AS1-transfected cells was determined by CCK-8 assay. C and D, Transwell assays were performed to confirm the migration and invasion of the indicated $\mathrm{PC}$ a cell lines. $* \mathrm{P}<0.05$, $* * \mathrm{P}<0.01, * * * \mathrm{P}<0.001$ 

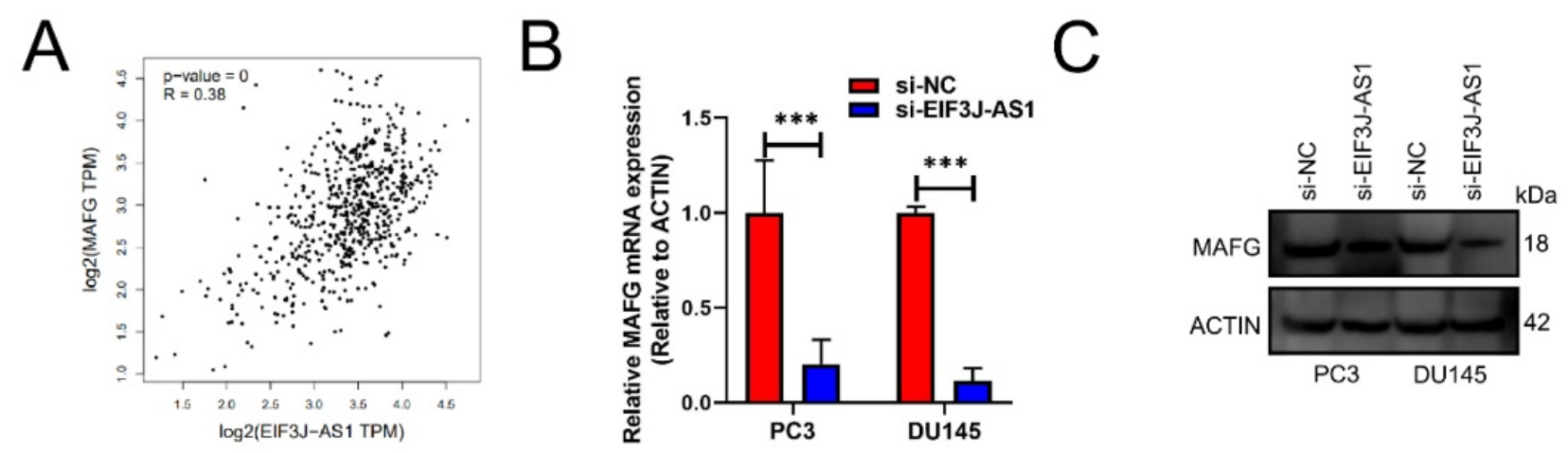

D
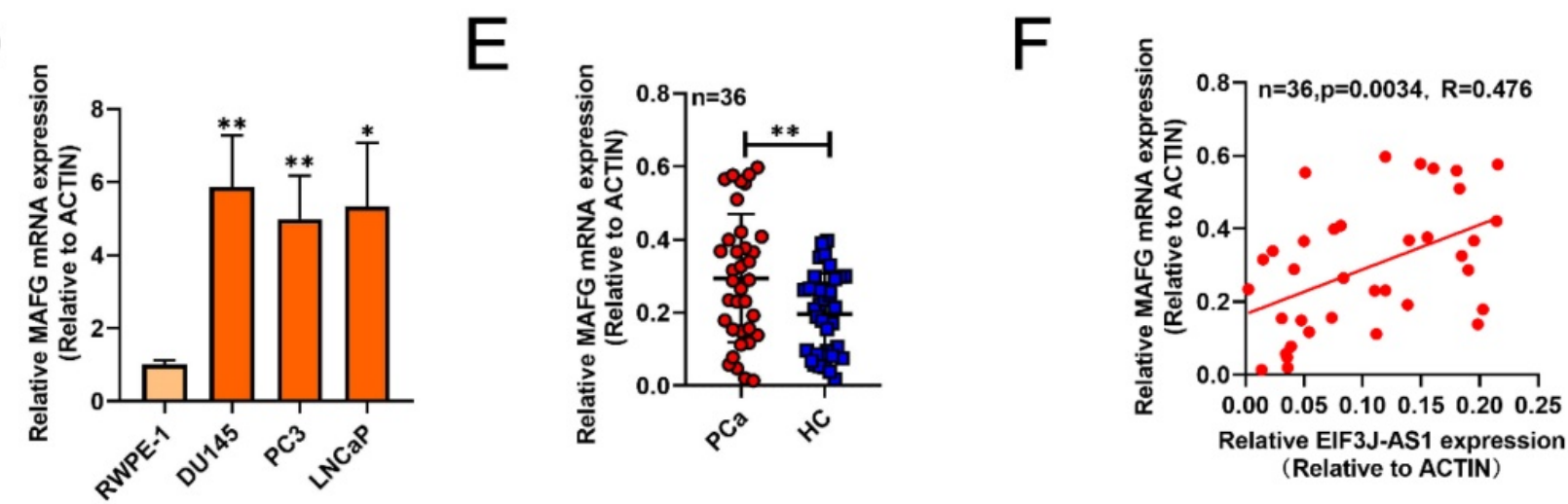

Figure 3. EIF3J-ASI is positively correlated with MAFG in PCa. A, GEPIA assessed EIF3J-ASI, and MAFG was positively correlated in PCa tissues. B and C, MAFG mRNA and protein expression in PC-3 and DU145 cells transfected with si-El3J-ASI was evaluated by qRT-PCR and western blot assay. D, qRT-PCR was used to assess EIF3)-AS1 expression in PCa cells (DU145, PC3 and LNCaP) and the human normal prostate epithelial cell line RWPE-1. E, MAFG mRNA expression in clinical tissue was assessed by GRT-PCR. F, The correlation between MAFG expression and El33-AS1 expression in $36 \mathrm{PCa}$ tissues was evaluated by Pearson's correlation analysis. $* \mathrm{P}<0.05$, $* * \mathrm{P}<0.01, * * * \mathrm{P}$ $<0.001$.

\section{EIF3J-AS1 is positively correlated with MAFG in $\mathbf{P C a}$}

Subsequently, we used GEPIA databases (http://gepia.cancer-pku.cn/) to screen out that EIF3J-AS1 was positively correlated with MAFG in PCa tissues $(p<0.001, r=0.38)$ (Figure 3A). The mRNA and protein levels of MAFG also markedly decreased in PC3 and DU145 cells transected with sh-EIF3J-AS1 (Figure $3 \mathrm{~B}$ and C). MAFG mRNA was also upregulated in DU145, PC3 and LNCaP PCa cell lines compared with the normal prostate epithelial cell line RWPE-1 (Figure 3D). We also verified the positive correlation of MAFD and EIF3J-AS1 at the mRNA level in $36 \mathrm{PCa}$ tissues from Changhai Hospital Biobank. (Figure 3E-F). These results suggested that MAFG is upregulated in PCa tissues and cells and has a positive correlation with EIF3J-AS1.

\section{Knockdown of MAFG inhibits PCa cell proliferation and invasion in vitro}

We then explored the effect of MAFG on the malignant phenotype of prostate cancer cells. First, appropriate cellular models were used with PC3 and DU145 cell lines because of their relatively high expression of MAFG. We treated those cells with MAFG knockdown (Figure 4A and B). The CCK-8 assay showed that knockdown of MAFG remarkedly inhibited the proliferation of both PC3 and DU145 cells (Figure 4C). Moreover, MAFG knockdown groups also indicated decreased migration and invasion ability in PC3 and DU145 cells (Figure 4D and E). Collectively, results showed that MAFG acts as an oncogene in PCa.

\section{EIF3J-ASI enhances PCa progression by upregulating MAFG}

Finally, we explored whether EIF3J-AS1 enhanced PCa cell growth by regulating MAFG by conducting rescue assays. We increased MAFG in PCa cells with EIF3J-AS1 knockdown (Figure 5A). The results of CCK-8 assays revealed that depleted EIF3J-AS1 markedly restrained the proliferation of PCa cells, but the transfection of LV-MAFG mitigated this effect (Figure 5B). In addition, increased MAFG countervailed the si-EIF3J-AS1-mediated alleviation of migration and invasion (Figure 5C and D). Thus, EIF3J-AS1 enhances PCa progression by upregulating MAFG.

\section{Discussion}

In recent years, an increasing number of studies have shown that the dysregulation of lncRNAs, such 
as lncRNA MALAT1 and DRAIC, plays important roles in the initiation and development of $\mathrm{PCa}[17-20]$. LncRNA EIF3J-AS1 was found to function as an oncogene in different kinds of cancer $[8,10]$ and to induce chemoresistance[21]. However, the role of EIF3J-AS1 has not been described with regard to PCa. In this study, we showed that EIF3J-AS1 was highly expressed in PCa tissues and cells, and knockdown of EIF3J-AS1 inhibited PCa cell proliferation, migration and invasion. These results described the driver of EIF3J-AS1 in PCa malignant progression. Thus, we further focused on how EIF3J-AS1 exerts its effects on PCa cells.

Previous studies showed that EIF3J-AS1 can exert its cancer-promoting effects by upregulating the expression of target genes, such as AKT1, YAP1 and ANXA11[8-10], via competing endogenous RNA[22, 23]. In this study, according to bioinformatics analysis showed that EIF3J-AS1 and MAFG were positively correlated in PCa tissues. MAFG is a bZIP-type transcriptional regulator that belongs to the small MAF (sMAF) protein family $[14,24]$. Previous studies showed that MAFG contributed to the malignant progression of cholangiocarcinoma, colorectal cancers and chemotherapy resistance [15, 25], acting as an oncoprotein. Even in hepatocellular cancer, a study showed that MAFG mRNA levels are at least 2-fold higher than those in adjacent nontumorous tissues in approximately half of clinical specimens [15]. However, the clinical significance and biological role of MAFG in PCa remain unknown. Further qRT-PCR experiments also validated that MAFG was significantly upregulated in PC tissues and cell lines. In addition, downregulation of MAFG in PCa cells resulted in the inhibition of proliferation and suppressed migration and invasion. In terms of preliminary mechanistic research, we found that downregulation of EIF3J-AS1 resulted in downregulated expression of MAFG in PCa cells. Rescue assays also showed that MAFG was involved in EIF3J-AS1-mediated PCa cell proliferation, migration and invasion. Thus, in this study, we provided evidence for a novel link between MAFG and EIF3J-AS1 in prostate cancer.

Thus, our study demonstrated that EIF3J-AS1/MAFG may play a key role in facilitating PCa progression and may serve as diagnostic biomarkers and potential therapeutic targets in PCa. However, the clear molecular mechanisms by which EIF3J-AS1 regulates MAFG upregulation and MAFG enhances cell growth and invasion in PCa must be further investigated in future research.

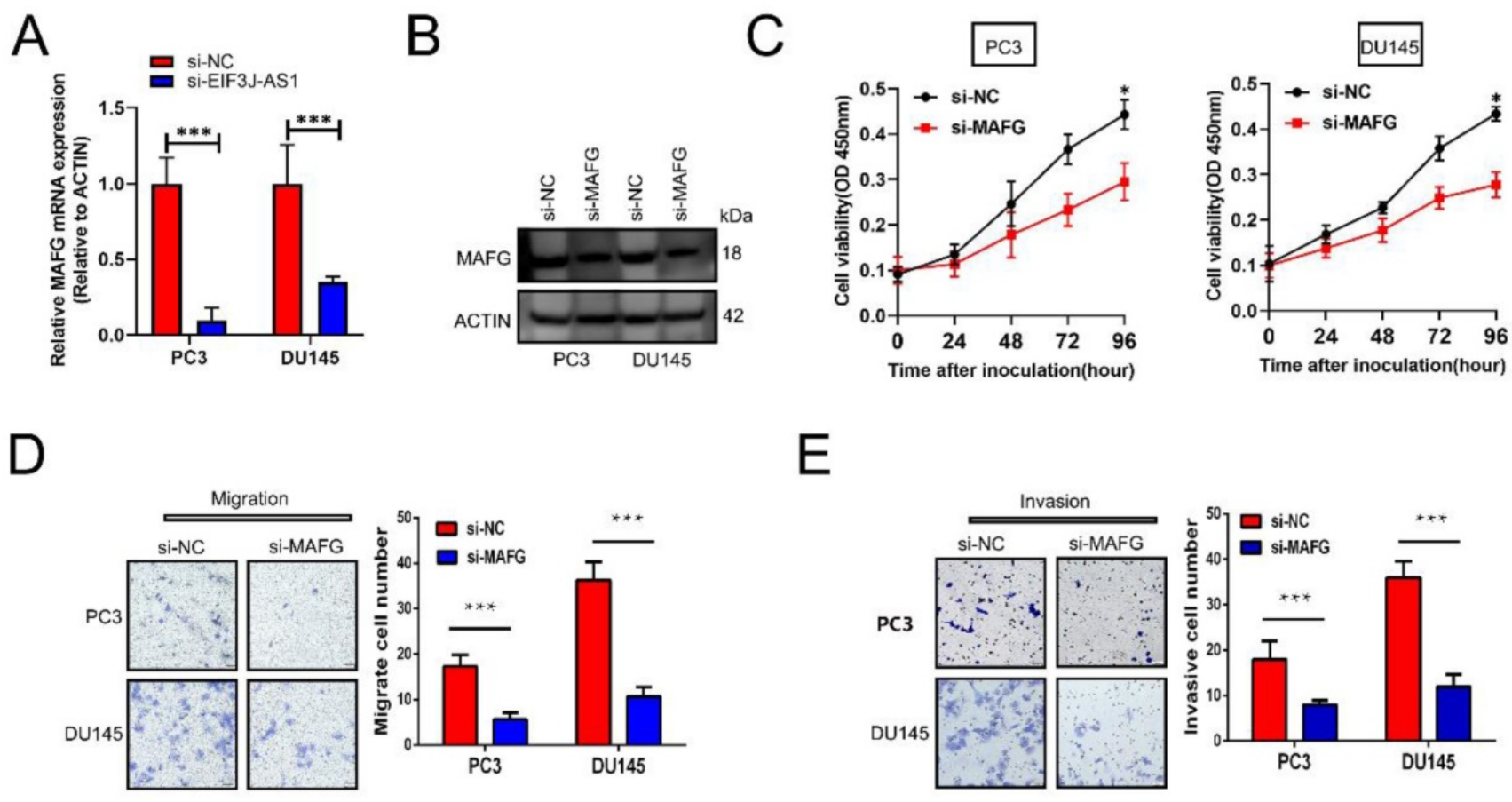

Figure 4. Knockdown of MAFG inhibits PCa cell proliferation and invasion in vitro. $A$ and $B, q R T-P C R$ and WB assays assessed MAFG mRNA and protein expression in the indicated treated PCa cell lines. C, The proliferation of the indicated PCa cells was determined by CCK-8 assay. D, The migration of the indicated PCa cells was determined by Transwell assay without Matrigel-coated membranes. E, The invasion of the indicated PCa cells was determined by Transwell assay with Matrigel-coated membranes. *P $<0.05$, $* * \mathrm{P}<0.01$, **** $\mathrm{P}<0.001$. 

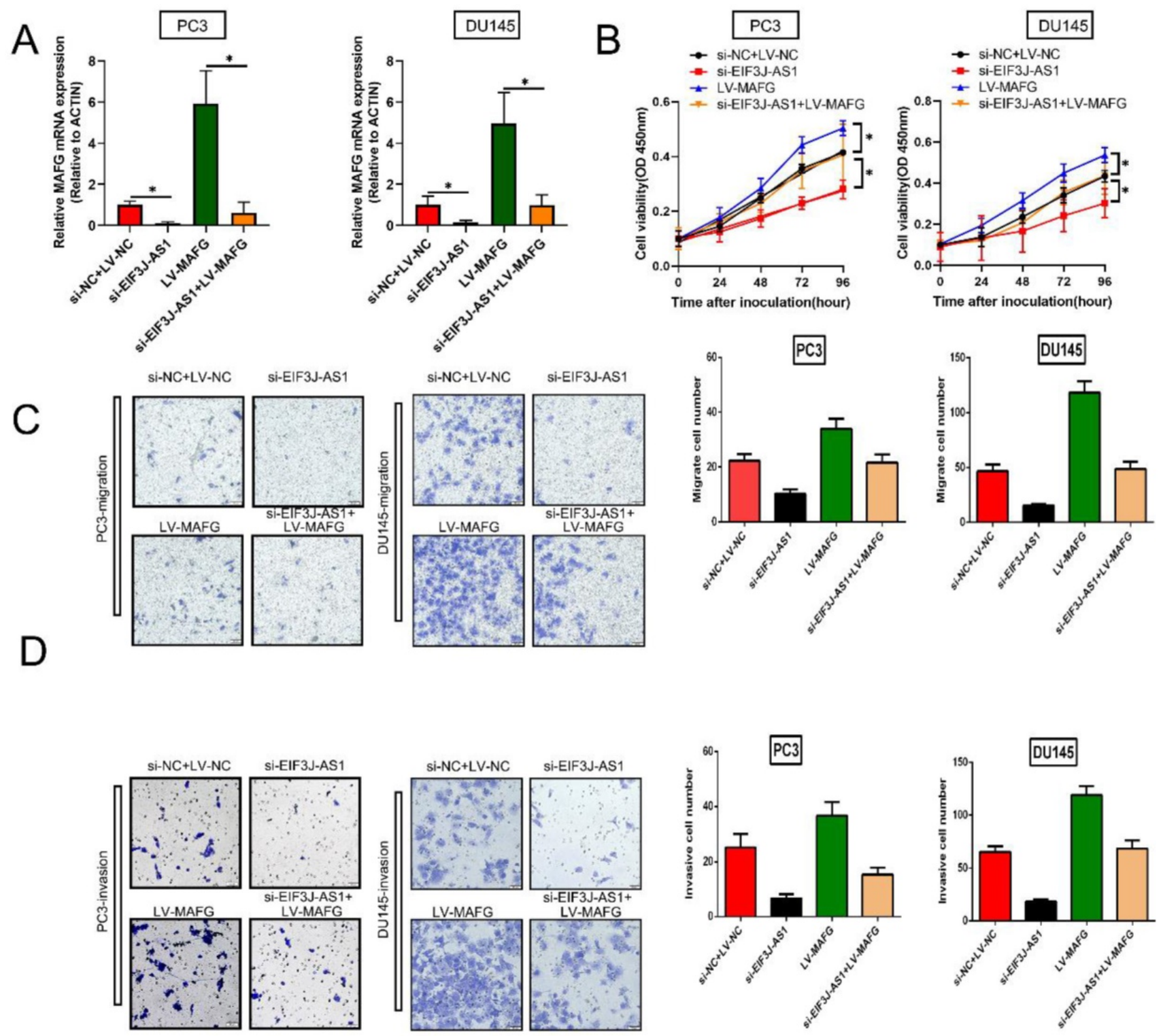

Figure 5. EIF3J-ASI enhances PCa progression by upregulating MAFG A. QRT-PCR assays assessed MAFG mRNA expression in the indicated treated PC cell lines. B, Proliferation in the indicated PCa cells was determined by CCK-8 assay. C, The migration of the indicated PCa cells was determined by Transwell assay without Matrigel-coated membranes. D, The invasion of the indicated PCa cells was determined by Transwell assay with Matrigel-coated membranes. $* \mathrm{P}<0.05, * * \mathrm{P}<0.01, * * * \mathrm{P}<0.001$

\section{Acknowledgements}

This study was supported by the Natural Science Foundation of Shanghai (13ZR1414300).

\section{Competing Interests}

The authors have declared that no competing interest exists.

\section{References}

1. Torre LA, Bray F, Siegel RL, Ferlay J, Lortet-Tieulent J, Jemal A: Global cancer statistics, 2012. CA Cancer J Clin 2015, 65(2): 87-108.

2. Preston MA, Batista JL, Wilson KM, Carlsson SV, Gerke T, Sjoberg DD, Dahl DM, Sesso HD, Feldman AS: Baseline Prostate-Specific Antigen Levels in Midlife Predict Lethal Prostate Cancer. J Clin Oncol 2016, 34(23): 2705-11.

3. Rahbar K, Afshar-Oromieh A, Jadvar H, Ahmadzadehfar H: PSMA Theranostics: Current Status and Future Directions. Mol Imaging 2018, 17: 1536012118776068 .
4. Abida W, Cyrta J, Heller G, Prandi D, Armenia J, Coleman I, Cieslik M, Benelli M, Robinson D, Van Allen EM: Genomic correlates of clinical outcome in advanced prostate cancer. Proc Natl Acad Sci U S A 2019, 116(23): 11428-11436.

5. Kaur H, Samarska I, Lu J, Faisal F, Maughan BL, Murali S, Asrani K, Alshalalfa M, Antonarakis ES, Epstein JI: Neuroendocrine differentiation in usual-type prostatic adenocarcinoma: Molecular characterization and clinical significance. Prostate 2020, 80(12): 1012-1023.

6. Huarte M: The emerging role of IncRNAs in cancer. Nat Med 2015, 21(11): 1253-61.

7. Lin C, Yang L: Long Non-coding RNA in Cancer: Wiring Signaling Circuitry. Trends Cell Biol 2018, 28(4): 287-301.

8. Liu D, Zhang H, Cong J, Cui M, Ma M, Zhang F, Sun H, Chen C: H3K27 acetylation-induced lncRNA EIF3J-AS1 improved proliferation and impeded apoptosis of colorectal cancer through miR-3163/YAP1 axis. J Cell Biochem 2020, 121(2): 1923-1933.

9. Qi J, Wang Z, Zhao Z, Liu L: EIF3J-AS1 promotes glioma cell growth via up-regulating ANXA11 through sponging miR-1343-3p. Cancer Cell Int 2020, 20: 428 .

10. Wei WT, Wang L, Liang JX, Wang JF, Li Q, Zeng J: LncRNA EIF3J-AS1 enhanced esophageal cancer invasion via regulating AKT1 expression through sponging miR-373-3p. Sci Rep 2020, 10(1): 13969.

11. Bartonicek N, Maag JL, Dinger ME: Long noncoding RNAs in cancer: mechanisms of action and technological advancements. Mol Cancer 2016, 15(1): 43. 
12. Statello L, Guo CJ, Chen LL, Huarte M: Gene regulation by long non-coding RNAs and its biological functions. Nat Rev Mol Cell Biol 2021, 22(2): 96-118.

13. Dykes IM, Emanueli C: Transcriptional and Post-transcriptional Gene Regulation by Long Non-coding RNA. Genomics Proteomics Bioinformatics 2017, 15(3): 177-186.

14. Motohashi H, Katsuoka F, Miyoshi C, Uchimura Y, Saitoh H, Francastel C, Engel JD, Yamamoto M: MafG sumoylation is required for active transcriptional repression. Mol Cell Biol 2006, 26(12): 4652-63.

15. Liu T, Yang H, Fan W, Tu J, Li TWH, Wang J, Shen H, Yang J, Xiong T, Steggerda J, Liu Z, Noureddin M, Maldonado SS, Annamalai A, Seki E, Mato JM, Lu SC: Mechanisms of MAFG Dysregulation in Cholestatic Liver Injury and Development of Liver Cancer. Gastroenterology 2018, 155(2): 557-571.e14.

16. Fang M, Hutchinson L, Deng A, Green MR: Common BRAF(V600E)-directed pathway mediates widespread epigenetic silencing in colorectal cancer and melanoma. Proc Natl Acad Sci U S A 2016, 113(5): 1250-5.

17. Ren S, Liu Y, Xu W, Sun Y, Lu J, Wang F, Wei M, Shen J, Hou J, Gao X, Xu C, Huang J, Zhao Y, Sun Y: Long noncoding RNA MALAT-1 is a new potential therapeutic target for castration resistant prostate cancer. J Urol 2013, 190(6): 2278-87.

18. Wang F, Ren S, Chen R, Lu J, Shi X, Zhu Y, Zhang W, Jing T, Zhang C, Shen J, Xu C, Wang $\mathrm{H}$, Wang $\mathrm{H}$, Wang $\mathrm{Y}$, Liu B, Li Y, Fang Z, Guo F, Qiao M, Wu C, Wei Q, Xu D, Shen D, Lu X, Gao X, Hou J, Sun Y: Development and prospective multicenter evaluation of the long noncoding RNA MALAT-1 as a diagnostic urinary biomarker for prostate cancer. Oncotarget 2014, 5(22): 11091-102.

19. Luo J, Wang K, Yeh S, Sun Y, Liang L, Xiao Y, Xu W, Niu Y, Cheng L, Maity $\mathrm{SN}$, Jiang $\mathrm{R}$, Chang C: LncRNA-p21 alters the antiandrogen enzalutamide-induced prostate cancer neuroendocrine differentiation via modulating via modulating the EZH2/STAT3 signaling. Nat Commun 2019, 10(1): 2571.

20. Saha S, Kiran M, Kuscu C, Chatrath A, Wotton D, Mayo MW, Dutta A: Long Noncoding RNA DRAIC Inhibits Prostate Cancer Progression by Interacting with IKK to Inhibit NF-KB Activation. Cancer Res 2020, 80(5): 950-963.

21. Luo Y, Zheng S, Wu Q, Wu J, Zhou R, Wang C, Wu Z, Rong X, Huang N, Sun L, Bin J, Liao Y, Shi M, Liao W: Long noncoding RNA (lncRNA) EIF3J-DT induces chemoresistance of gastric cancer via autophagy activation. Autophagy 2021,25: 1-19.

22. Yuan JH, Yang F, Wang F, Ma JZ, Guo YJ, Tao OF, Liu F, Pan W, Wang TT, Zhou CC, Wang SB, Wang YZ, Yang Y, Yang N, Zhou WP, Yang GS, Sun SH: A long noncoding RNA activated by TGF- $\beta$ promotes the invasion-metastasis cascade in hepatocellular carcinoma. Cancer Cell 2014, 25(5): 666-81.

23. Chan JJ, Tay Y: Noncoding RNA:RNA Regulatory Networks in Cancer. Int J Mol Sci 2018, 19(5):1310.

24. Caggiano R, Cattaneo F, Moltedo O, Esposito G, Perrino C, Trimarco B, Ammendola R, Faraonio R: miR-128 Is Implicated in Stress Responses by Targeting MAFG in Skeletal Muscle Cells. Oxid Med Cell Longev 2017, 2017: 9308310.

25. Vera-Puente $O$, Rodriguez-Antolin $C$, Salgado-Figueroa A, Michalska $P$, Pernia O, Reid BM, Rosas R, Garcia-Guede A, Sacristán S, Jimenez J, Esteban-Rodriguez I, Martin ME, Sellers TA, León R, Gonzalez VM, De Castro $\mathrm{J}$, Ibanez de Caceres I: MAFG is a potential therapeutic target to restore chemosensitivity in cisplatin-resistant cancer cells by increasing reactive oxygen species. Transl Res 2018, 200: 1-17. 